\section{ORIGINAL RESEARCH}

\author{
S. Gallas \\ A.C. Januel \\ A. Pasco \\ J. Drouineau \\ J. Gabrillargues \\ A. Gaston \\ C. Cognard
}

D. Herbreteau

\title{
Long-Term Follow-Up of 1036 Cerebral Aneurysms Treated by Bare Coils: A Multicentric Cohort Treated between 1998 and 2003
}

\begin{abstract}
BACKGROUND AND PURPOSE: The endovascular treatment (EVT) of cerebral aneurysms has experienced a revolution since 1991 with the introduction of platinum coil technology. During the past 10 years, there has been significant study of the feasibility of this technique, and clinical results of EVT have been published. The long-term durability of Guglielmi detachable coil (GDC) embolization of cerebral aneurysms still remains unknown. The purpose of this study was to evaluate the stability of anatomic occlusion of aneurysms and to assess the rate of recanalization and retreatment of these aneurysms.
\end{abstract}

MATERIALS AND METHODS: Between January 1998 and December 2003, 1036 aneurysms (804 ruptured and 232 nonruptured) were treated consecutively with GDC coils in 5 neuroradiology centers. Procedural feasibility, acute angiographic occlusion results, morbidity, and mortality associated with this technique were assessed. All patients were regularly followed by digital subtraction angiography and MR imaging each year after treatment.

RESULTS: Initial acute angiographic results in 1036 aneurysms demonstrated total occlusion in 731 patients $(70.5 \%)$, subtotal occlusion in $252(24.3 \%)$, incomplete occlusion in $20(1.9 \%)$, and failures in $33 \%(3.3 \%)$ aneurysms. A remodeling technique was used in $10 \%$. A second procedure was performed for 72 aneurysms (7\%). The total aneurysm follow-up time was 49,923 months (4160.25 aneurysm-years). The retreatment period was either in the months following initial treatment in aneurysms incompletely occluded or in years due to recanalization or de novo aneurysms. Fewer than 5 patients rebled during 10 years of follow-up. Long-term follow-up angiograms were obtained in 899 aneurysms, with 646 total, 230 subtotal, and 23 incomplete results.

CONCLUSIONS: Long-term follow-up of cerebral aneurysms is necessary to depict recanalization. Only $7 \%$ of the aneurysms were retreated. Use of bare coils gives a good long-term level of occlusion.

E ndovascular embolization with detachable coils has become an established technique for the treatment of patients with intracranial aneurysms. Endovascular treatment (EVT) of cerebral aneurysms experienced a revolution with the introduction of platinum coil technology in 1991. ${ }^{1,2}$ During the past 10 years, there has been significant study of the feasibility of this technique, and clinical results of EVT have been published by endovascular specialists worldwide. ${ }^{2-8}$ In 2002, results of the International Subarachnoid Aneurysm Trial (ISAT) demonstrated the clinical superiority of EVT over standard surgical treatment of ruptured intracranial aneurysms. ${ }^{3}$ Some articles have been published pertaining to the stability of occlusions treated with detachable coils and the efficacy of providing protection against growth or regrowth of the aneurysm and consequent bleeding. ${ }^{4-16}$ Long-term follow-up and the rate of recanalization due to coil compaction, typically caused by unfavorable morphologic characteristics

Received March 20, 2009; accepted after revision May 19.

From the Department of Neuroradiology (S.G., D.H.), University François Rabelais, Centre Hospitalier Universitaire Bretonneau, Tours, France; Department of Neuroradiology (A.C.J., C.C.), Centre Hospitalier de Toulouse, Hôpital Purpan, Toulouse, France; Department of Radiology (A.P.), Centre Hospitalier Universitaire d'Angers, Angers, France; Department of Radiology A (J.D.), Centre Hospitalier Universitaire de Poitiers, Poitiers, France; Department of Radiology (J.G.), Centre Hospitalier Universitaire de Clermont Ferrrand, Clermont Ferrand, France; and Department of Neuroradiology (S.G., A.G.), Hopital Henri Mondor, Creteil, France.

Please address correspondence to Sophie Gallas, MD, Department of Neuroradiology, Centre Hospitalier Henri Mondor, Creteil Cedex, France; e-mail: sophie_gallas@yahoo.fr and sophie.gallas@hmn.aphp.fr

DOl 10.3174/ajnr.A1744 such as lesion and neck size, remain a disadvantage compared with surgical treatment.

The aim of this study was to better assess the long-term durability of Guglielmi detachable coil (GDC) (Boston Scientific/Neurovascular, Fremont, Calif) treatment of cerebral aneurysms and the rate of required second treatments.

\section{Materials and Methods}

\section{Patients}

This study was approved by the institutional review boards of 5 participating hospitals in France. Since 1998, all patients with ruptured or unruptured cerebral aneurysms who underwent endovascular treatment were recruited and recorded in a data base. All treatments, retreatments, and follow-ups for each patient were consecutively collected. Every patient from each center was followed up by the representative interventional physician, and we conducted annual meetings to collect and review all cases. From 1998 to 2003, 929 patients were enrolled in this study, with a total of 1036 aneurysms treated. Feasibility, morbidity-mortality, and initial results were previously published. ${ }^{13,14}$ We present the long-term results of occlusion after follow-up (mean, 66 months; range, 3-10 years).

\section{Patient Characteristics}

From January 1998 to December 2003, 1036 cerebral aneurysms were considered for endovascular treatment with detachable coils at 5 neuroradiologic centers. We collected all procedures, initial and second treatments after follow-up, to obtain 1108 total procedures. Of the 1036 cerebral aneurysms, 804 were initially ruptured; 232 were unruptured; and 72 were second procedures (Table 1). Clinical presen- 


\begin{tabular}{lccccc}
\hline Table 1: Number of cerebral aneurysms seen at each institution \\
\hline & \multicolumn{5}{c}{ Department of Neuroradiology } \\
\cline { 2 - 6 } & Clermont \\
Aneurysms & Angers & Ferrand & Poitiers & Toulouse & Tours \\
\hline No. ruptured & 157 & 165 & 151 & 142 & 189 \\
No. unruptured & 36 & 65 & 36 & 35 & 60 \\
Total No. & 193 & 230 & 187 & 177 & 249 \\
\hline
\end{tabular}

\begin{tabular}{lcc}
\hline Table 2: Location of cerebral aneurysms & & \\
\hline Location & No. & $\%$ \\
\hline Anterior circulation & 977 & 88 \\
Anterior communicating artery/anterior artery & 409 & 37 \\
Pericallosal artery & 30 & 2.7 \\
Posterior communicating artery & 191 & 17 \\
Carotid/ophthalmic artery & 183 & 16.5 \\
Middle cerebral artery & 143 & 12.8 \\
Internal carotid artery & 21 & 2 \\
Posterior circulation* & 131 & 11 \\
Vertebrobasilar artery & 65 & 5.7 \\
Posterior inferior cerebellar artery & 40 & 3.6 \\
Anterior inferior cerebellar artery & 8 & 0.2 \\
Posterior cerebral artery & 18 & 1.5 \\
\hline
\end{tabular}

* breakdown of aneurysms treated.

tation, symptoms, aneurysm location, size and shape of the aneurysm sac, and patient age were recorded. Women represented $65 \%$ of the treated population and men, $35 \%$, with a mean age of 52.38 years (median, 53 years; range, $18-84$ years).

All patients with subarachnoid hemorrhage underwent cranial CT scanning and were examined by a neurosurgeon and neuroanesthetist. External CSF drainage was initiated before endovascular treatment in patients presenting with hydrocephalus. Decision to treat was made by consensus by the referring neurosurgeon and the interventional physician. The severity of subarachnoid hemorrhage was clinically assessed at the time of admission by using the Hunt and Hess $(\mathrm{HH})$ grading scale ${ }^{11}$ and the Fisher scale. For the HH scale, $18 \%$ of patients were admitted with clinical grade I $(n=159) ; 40 \%$, with grade II ( $n=312) ; 16 \%$, with grade III $(n=136)$; and $26 \%$, with grade IV $(n=197)$. For the Fisher scale, the distribution of the population was as follows: $10 \%$, scale I ( $n=97) ; 40 \%$, scale II $(n=314) ; 20 \%$, scale III $(n=180)$; and $30 \%$ scale IV $(n=213)$.

We conferred with all patients with unruptured aneurysms before treatment to explain the risks and objectives of endovascular treatment. These 232 aneurysms were incidentally discovered during imaging studies performed for clinical reasons unrelated to the presence of an aneurysm.

The various locations of the treated aneurysms are detailed in Table 2. Aneurysms located in the anterior circulation represented $88 \%$ of all aneurysms treated. All aneurysm-dimension measurements were made on diagnostic angiograms. Small aneurysms measuring $<5 \mathrm{~mm}$ represented $28 \%$ of all cases. Aneurysms measuring between 5 and $9 \mathrm{~mm}$ represented $55.3 \%$ of all cases, $9.4 \%$ of all aneurysms measured between 10 and $14 \mathrm{~mm}$, and 5.3\% of aneurysms measured between 15 and $19 \mathrm{~mm}$. Aneurysms $<10 \mathrm{~mm}$ represented $83 \%$ of all aneurysms in this series. Twenty aneurysms were defined as giant, measuring $>20 \mathrm{~mm}$. The balloon remodeling technique was used in the treatment of 109 aneurysms (10\%).

\section{Coiling Procedures}

Endovascular treatment was performed by 5 qualified physicians, each with a minimum of 5 years' experience performing EVT with
GDC before initiation of this study. Endovascular management for all patients treated as part of this study series was similar in technique, approach, and follow-up. Protocol of treatment, device used, and follow-up were determined by consensus before the beginning of this study. All aneurysms were embolized with bare platinum GDC coils. No other bare platinum coils or polymer-coated coils were used in our centers during the course of this study. All patients were treated under general anesthesia. Systematic heparinization was performed after placement of the arterial introducer sheath, according to our embolization protocol; $3000 \mathrm{IU}$ of heparin was administered as an intravenous bolus injection, followed by an additional 1000 IU per hour. Patients with unruptured aneurysms received antiplatelet medication before endovascular treatment and for 1 month following treatment to avoid thromboembolic events.

In all cases, coil size and length, coil shape, and the total number of coils used were recorded. No intracranial stents and no modified coils were used at this time. All procedural complications were also recorded. Postprocedure, all patients were treated in the neurosurgery intensive care unit, those with ruptured aneurysms for at least 10 days. Patients with poor clinical grades were observed in the intensive care unit until stabilization of hemodynamic parameters was achieved. Anticoagulation was routinely continued for the first 48 hours postprocedure.

\section{Angiographic Results and Follow-Up}

Initial angiographic results of coil occlusion were classified as complete, near-complete, or incomplete by using the modified Raymond classification scale. ${ }^{5-8}$ Aneurysm occlusion at the end of the procedure was considered to be "complete" when the aneurysmal sac and neck were packed and no contrast filling the sac was detected by angiography. Occlusion was considered "near-complete" or "subtotal" when the sac was occluded but a neck remnant was observed. "Incomplete occlusion" was defined as an aneurysm having loose packing and partial opacification of the aneurysm sac. The distinction between small recurrences (small change in packing) and full recanalization was determined by the performing physician. Recanalization, as a consequence of coil compaction due to high arterial blood flow or aneurysm growth, was considered to require a second treatment because of the high risk of rupture.

By consensus, all treating physicians decided that each patient would undergo follow-up angiography by digital substraction angiography (DSA) and MR imaging at 3 months, 12 months, and subsequent annual examinations. Multiple projections with selective injections served to define any residual lesions. These projections were compared with the initial results of occlusion. In each center, the treating physician was responsible for follow-up and review of each patient after DSA and MR imaging.

Each year after treatment, patients were followed by MR imaging for 5 years. In the case of discordance of results or in patients with a potential high risk for coil compaction, additional follow-up angiography was performed. After 5 years, if stability of occlusion was observed, the patient was evaluated by the practitioner and assessed with MR imaging every 2 years. DSA was performed in cases of coil compaction or modification of packing. After 10 years, patients were evaluated via MR imaging and clinical examination.

The final MR imaging and/or MR angiography (MRA) follow-up examinations (axial source images, maximum-intensity projections, and volume-rendered images) for every patient were evaluated and compared with the previous imaging studies by 2 experienced neuroradiologists, independent of the treating physician. 
number of aneurysms retreats and proportion of retreats to follow-up.

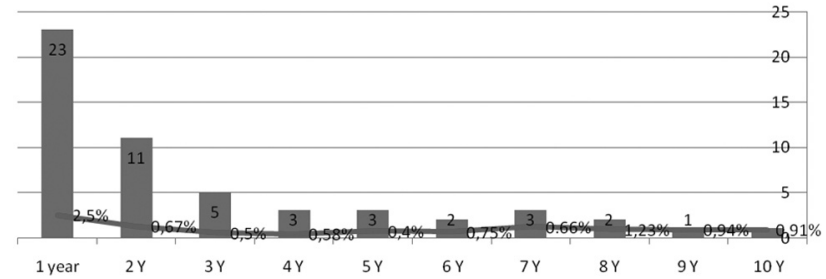

Fig 1. Graph shows the number of aneurysms retreated for 10 years' follow-up. Columns indicate new treatments; lines are the proportion of aneurysms retreated.

\section{Results}

Between January 1, 1998, and December 31, 2003, angiographic analysis and clinical data were obtained for a total of 1108 GDC-treated intradural saccular aneurysms in 929 patients. A total of 1036 intradural aneurysms underwent a singular EVT with GDC coils, of which 804 were ruptured (73\%) and 232 were unruptured (27\%), and 72 aneurysms underwent a second treatment for a total of 1108 procedures.

Feasibility and Efficacy of Treatment. In 33 aneurysms (3.5\%), treatment failed due to the shape of the aneurysm or instability of the coil on deployment. No coils could be safely placed in any of these aneurysms. Twenty-eight aneurysms were catheterized, but coil deployment failed due to coil instability inside the aneurysmal sac, indicating a high risk of migration. If possible, patients with a hemorrhage $(n=18)$ were subsequently referred for surgical clipping. Asymptomatic patients ( $n=15$, with no ruptured aneurysms) were reprogrammed to have a new procedure to assess occlusion. Overall procedural feasibility of occlusion with GDC coils was $96.5 \%$.

Immediate Posttreatment Results. At the end of the initial procedure of the 1036 aneurysms treated, occlusion was classified as complete in 731 cases (70.5\%), subtotal in 252 cases (25\%), and incomplete in 20 cases (1.4\%). Seventy-two aneurysms underwent a second treatment (retreatment) during the follow-up: 20 due to initial incomplete occlusion and 52 with modification of the initial packing of the coils and regrowth of the aneurysm. In our population, the remodeling technique was used for 109 aneurysms, representing 10\% of all procedures.

Initial Mortality. Due to the severity of subarachnoid hemorrhage, vasospasm, or associated hematoma, 96 patients with 98 ruptured aneurysms died during the first 30 days after treatment. Overall mortality was $11.4 \%$, with vasospasm as the first etiology.

Only 1 patient died secondary to perforation of 1 unruptured aneurysm during the embolization procedure. This patient had a previous history of subarachnoid hemorrhage with a good outcome of treatment of the ruptured aneurysm. During embolization of the second unruptured aneurysm, a perforation and subsequent subarachnoid hemorrhage and hematoma occurred. The patient died 3 days after treatment due to the hematoma. A total of 99 aneurysms were not controlled due to death of the patient.

\section{Second Treatment}

Retreatment (Fig 1 and Table 3) was performed if there was a significant morphologic loss during angiographic follow-up

\begin{tabular}{|c|c|c|c|c|}
\hline Characteristic & $\begin{array}{l}<1 \mathrm{An}, \\
<12 \mathrm{Mo}\end{array}$ & $\begin{array}{c}1 \mathrm{An} \\
12-18 \mathrm{Mo}\end{array}$ & $18 \mathrm{Mo}-5 \mathrm{Yr}$ & $>5 \mathrm{yr}$ \\
\hline No. treated & 41 & 6 & 15 & 10 \\
\hline \multicolumn{5}{|l|}{ Lumen size } \\
\hline Small & 19 & 3 & 9 & 2 \\
\hline Medium & 16 & 1 & 4 & 7 \\
\hline Large & 6 & 2 & 2 & 1 \\
\hline \multicolumn{5}{|l|}{ Aneurysm location } \\
\hline ACA & 13 & 3 & 5 & 4 \\
\hline PCA & 17 & 1 & 3 & 3 \\
\hline ICA & 4 & 1 & 2 & 1 \\
\hline MCA & 2 & & 3 & \\
\hline Posterior artery & 5 & 1 & 2 & 2 \\
\hline \multicolumn{5}{|l|}{ Initial clinical conditions } \\
\hline Initial hemmorhage & 31 & 5 & 11 & 7 \\
\hline Unruptured & 10 & 1 & 4 & 3 \\
\hline
\end{tabular}

Note:-An indicates aneurysm; ACA, anterior cerebral artery; PCA, posterior communicating artery; ICA, internal carotid artery; MCA, middle cerebral artery.

or if the initial result was unsatisfactory. Sixty-two of 1036 aneurysms were retreated one or more times for a total of 72 new procedures (53 aneurysms were retreated once; 8 aneurysms twice and 1 aneurysm three times during the follow-up period). Of the retreated cerebral aneurysms, 46 were initially ruptured (74\%) and 16 were unruptured. For 5 patients, endovascular retreatment was insufficient and the patient was inevitably referred for open surgical clipping. We recorded all characteristics of aneurysm retreatment, such as location, size, initial result and delay between the 2 procedures, result obtained after the second procedure, and result at final followup. Location of aneurysms retreated was as follows: 61, in the anterior circulation; and 11, at the posterior artery (basilar artery or posterior inferior cerebellar artery).

The most usual locations requiring retreatment were the anterior communicating artery and the posterior communicating artery. The size of aneurysms was as follows: 33 were $<5$ $\mathrm{mm}$ in diameter, 28 were between 5 and $10 \mathrm{~mm}$, 5 were $>10$ and $<15 \mathrm{~mm}$ in diameter, and 6 were giant aneurysms. The median aneurysmal sac size was $7.4 \mathrm{~mm}$ (range, $4-19 \mathrm{~mm}$ ).

The time between the first procedure and the second treatment was also recorded. The midpoint delay between the 2 procedures was 702 days (23 months), with a median of 10 months (range, 10-3362 days). Two groups of patients were individualized. For 47 aneurysms (66\%), initial treatment was insufficient and an important early modification of the initial packing of the coils was observed during follow-up. These patients were rapidly retreated within the first 18 months (41 during the first year and 6 between 12 and 18 months).

For the second group of patients, modification of packing was not observed until 5-6 years after the initial procedure. These aneurysms were in the same location as the initial aneurysm, (no modification of packing occurred) similar to the occurrence of a de novo aneurysm. In total, 15 aneurysms were retreated between 18 months and 5 years after treatment, and 10 aneurysms were retreated after 5 years (Table 3 ).

For 5 aneurysms $(8 \%, 5 / 62)$, the second treatment failed or was not effective and a new recanalization appeared. For these very unstable aneurysms, an open surgical treatment was proposed after discussion with the neurosurgeon. Surgery was successful in 4 patients, and for 1 patient, surgery was not 


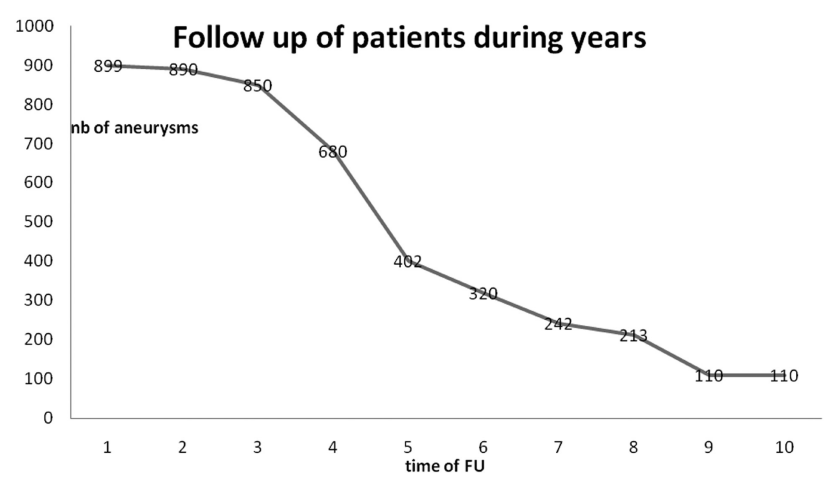

Fig 2. Graph shows follow-up (FU) of patients for 10 years

completed and a new recanalization, or de novo aneurysm, was observed. This patient rebled after failure of surgery and was retreated by EVT in the emergency department with a poor clinical outcome.

Rebleeding. Five patients (5/969, 0.5\%) rebled during follow-up. Three patients were retreated in the emergency department: 1 patient underwent surgery in another center and 1 patient died. All of these patients were initially treated for a ruptured aneurysm. No patients with unruptured aneurysms bled.

Follow-Up. The aim of this prospective study was to assess the long-term follow-up of the treated aneurysms. Initially all patients were to be followed up annually. Considering the patients who died because of the severity of the initial hemorrhage ( $n=97$ patients with 99 aneurysms) and patients who were lost to follow-up ( $n=70$ patients with 75 aneurysms), we reviewed 762 patients with 899 aneurysms at a minimum of 1 time posttreatment. During follow-up, unrelated death was reported in 11 patients, due to cancer in 5 patients, cardiovascular disease in 3 patients, and old age in 3 patients.

Time of Follow-Up. We followed up 899 aneurysms at 1 year, 890 aneurysms 2 years after treatment (99\%), 850 (94\%) aneurysms at 3 years after treatment, and 402 aneurysms (44\%) 5 years after initial treatment. A total of 213 aneurysms were assessed at 8 years $(23.6 \%)$ after treatment and 110 (12.2\%) were reviewed at 10 years. Total aneurysm follow-up time was 49,923 months (4160.25 aneurysm-years), with a mean of 55.6 months and a median of 53 months (Fig 2).

\section{Early and Final Results for 804 Ruptured Aneurysms}

A total of 804 ruptured aneurysms were included in this study. Initial occlusion results were complete for 559 aneurysms (69.5\%), subtotal for 210 aneurysms (26\%), and partial or incomplete for 17 aneurysms (2.1\%). EVT failed for 18 aneurysms $(2.1 \%)$. In this population, 98 aneurysms were not followed up because of the death of the patient secondary to complications of the initial hemorrhage, and 64 were lost to follow-up. A total of 624 ruptured aneurysms were followed up. The last angiographic results obtained for these 624 aneurysms were as follows: 450 were completely occluded (72\%), 160 had a neck remnant $(25.6 \%)$, and 14 (2.4\%) were incompletely occluded.

\section{Early and Final Results for 232 Unruptured Aneurysms}

A total of 232 unruptured aneurysms were considered for EVT in this study. Initial occlusion results were complete for 172

\begin{tabular}{lrrrrrrr}
\hline \multicolumn{7}{l}{ Table 4: Evolution of each initial class of occlusion } \\
\hline \multicolumn{7}{c}{ Initial Occlusion } \\
\cline { 2 - 7 } Final Occlusion & Complete & $\%$ & Subtotal & $\%$ & Incomplete & $\%$ & No. \\
\hline Complete & 502 & 69.6 & 92 & 36.5 & 2 & 10 & 596 \\
Subtotal & 99 & 13.5 & 105 & 41.7 & 6 & 30 & 210 \\
Incomplete & 10 & 1.4 & 6 & 2.4 & 7 & 35 & 23 \\
Death & 64 & 8.8 & 31 & 12.3 & 4 & 20 & 99 \\
Lost to follow-up & 56 & 7.7 & 18 & 7.1 & 1 & 5 & 75 \\
No. aneurysms & 731 & & 252 & & 20 & & 1003 \\
\hline
\end{tabular}

aneurysms (74.1\%); subtotal, for 42 aneurysms (18\%); and partial, for 3 aneurysms (1\%). EVT failed in 15 aneurysms $(6.4 \%)$. In this population of patients, 1 patient died after hemorrhage during the procedure and 11 with aneurysms were lost to follow-up. Final angiographic results were collected for 205 aneurysms: 146 aneurysms were completely occluded (71.2\%), 50 were subtotally occluded (24.3\%), and 9 $(4.3 \%)$ were incomplete at last follow-up.

\section{Results and Evolution of Occlusion for Initial Total, Subtotal, and Incomplete Occlusion}

Evolution of the 731 Aneurysms with Initial Total Occlusion. At the time of preparation of this article, $502(68.6 \%)$ completely occluded aneurysms remained stable, with no modification of the immediate posttreatment result at the final angiographic follow-up examination. A small recurrence was observed in $99(13.5 \%)$ patients. Coil compaction with a remnant was observed in 10 patients $(1.3 \%)$. A total of 20 aneurysms that were initially completely occluded underwent retreatment. Fifty-six patients died, and 64 patients were lost to follow-up.

Evolution of 252 Aneurysms with Subtotal Occlusion. In $105(41.6 \%)$ patients, no modification of occlusion from the initial treatment was noted. In 92 patients $(36.5 \%)$, there was a spontaneous thrombosis of the sac of the aneurysm resulting in a total occlusion. Eighteen patients were lost to follow-up $(18 / 252,7.1 \%)$ and 31 died $(31 / 252,12.3 \%)$. Six aneurysms had a small recanalization, and 28 aneurysms from this category were considered for a second treatment to improve the initial occlusion.

Evolution of 20 patients with Incomplete Initial Occlusion. Of the 20 initial incomplete occlusions, 7 patients underwent a second procedure to ameliorate coil packing in the sac, 8 aneurysms had spontaneous amelioration of packing due to thrombosis, 4 patients died, and 1 patient was never followed.

Stability of the Occlusion. We determined stability of occlusion when no change of initial coil packing appeared, amelioration when spontaneous intra-aneurysmal thrombosis occurred, and aggravation when an aneurysm was reopened. All controlled aneurysms were evaluated before any second procedures were performed (Table 4).

For all controlled aneurysms, before the second procedure, stability for occlusion was achieved for 614 aneurysms (74.1\%); amelioration, for 100 aneurysms (12.1\%); and aggravation (change of classification, loose packing), for 115 aneurysms $(13.8 \%)$.

Results of Last Angiographic Follow-Up for 899 Procedures. At the final available follow-up at the time of this writing, angiographic results, including the 62 aneurysms treated 


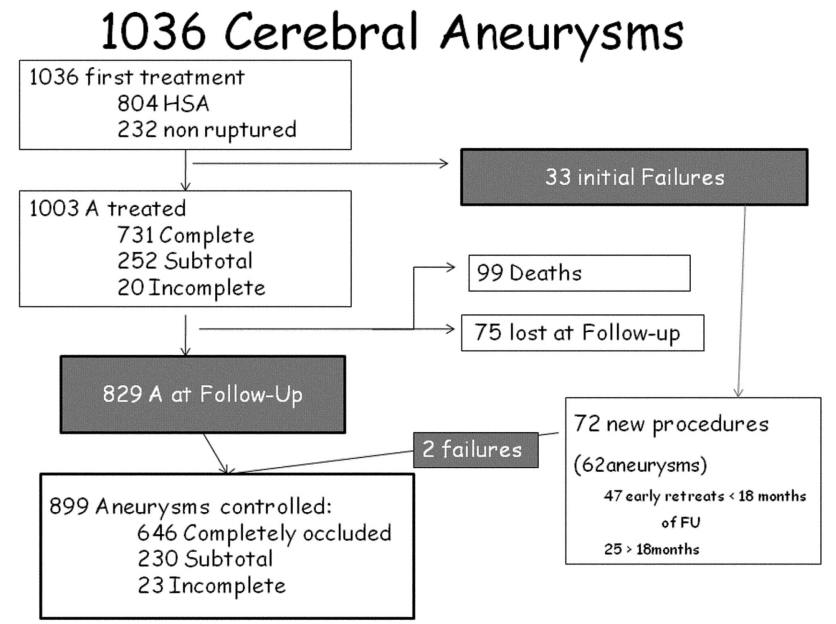

Fig 3. Consort flow chart shows 1036 aneurysmal procedures. HSA indicates XXX; $A$, aneurysms.

twice (72 new procedures), were as follows: 646 aneurysms were completely occluded (71.9\%), 230 aneurysms were subtotally occluded (25.6\%), and 23 aneurysms (2.5\%) were incompletely occluded (Fig 3).

\section{Discussion}

Endovascular embolization with detachable coils has become an established technique for the treatment of patients with intracranial aneurysms, confirmed by the results of the ISAT study. ${ }^{1-9}$ In many neuroradiologic centers, endovascular treatment is the first line for patients. The long-term follow-up of this technique, however, is largely unknown. This study was undertaken to analyze the clinical and morphologic long-term outcomes of cerebral aneurysms. There are very few series published with $>5$ years of results. Our multicentric series includes $>402$ aneurysms controlled at 5 years; 213 , at 8 years; and 110 aneurysms, at 10 years. These results are valid for assessing the stability of occlusion with bare coils. Protocol of follow-up is not well established and is inconsistent. Some physicians recommend follow-up for $\leq 2$ or 3 years posttreatment, whereas others will discontinue follow-up after 1 year posttreatment if coil occlusion is stable. ${ }^{12}$

Before the collection of data for this series, we decided to carry out controlled follow-up of all patients for at least 10 years, because EVT was a fairly new treatment technique and the long-term results were unknown. Since 1998, we consecutively and prospectively collected results on all endovascularly treated aneurysms from our 5 respective centers. Initial feasibility and acute outcome results for ruptured and unruptured aneurysms in this series have been published. ${ }^{10,13,14}$

All patients treated with an endovascular technique were sought and included those with berry aneurysms and large and giant aneurysms. Only dissecting vertebral aneurysms were excluded from this multicentric study because it is known that the evolution of these aneurysms differs from that of saccular aneurysms. During this collection period and before "stentassisted coiling" or development of modified coils, 75\% of all ruptured aneurysms were treated via EVT and 25\% were surgically treated. Difficult aneurysms, large aneurysms, or middle cerebral artery aneurysms were also often referred for surgical treatment. In our consecutive study, only $12.8 \%$ of middle cerebral artery aneurysms were coiled, and the balloon-assisted technique was used in $10 \%$ of all patients.

In 2009 , this technique is used in $>30 \%$ of all procedures. Middle cerebral artery aneurysms are also now frequently treated by an endovascular approach, with the use of a balloon or intracranial stent. ${ }^{15}$ Nevertheless, only ruptured aneurysms with an intracerebral hematoma still undergo surgery to evacuate the hematoma and clip the aneurysm at the same time.

Assessment of the stability of occlusion with time was important. In $74.1 \%$ of aneurysms, no modification of the initial angiographic result was observed, $12.1 \%$ demonstrated amelioration of the occlusion with spontaneous thrombosis, and $13.1 \%$ had aggravation. Sixty-two aneurysms (6\%) were retreated in 72 new procedures during long-term follow-up. The safety of endovascular treatment has been established, and our series can now provide insight into the long-term stability of occlusion beyond 5 years. The long-term clinical follow-up data demonstrated a very low rebleeding rate, $<1 \%$, which is in accordance with previous studies. In the report of Aikawa et al, ${ }^{12}$ the rate of rebleeding after endovascular treatment was $2.6 \%$, with an interval time between treatment and rebleeding of $<1$ year in $80 \%$. They proposed an initial follow-up within the first 2 months posttreatment, followed by 2 examinations in the first year and an annual follow-up thereafter. Long-term follow-up is necessary, especially in patients who may be at risk for rebleeding and in patients who bled precociously during the first 18 months.

We did not have an evolution of patients whose aneurysms were clipped during this same period. In the Cerebral Aneurysm Rerupture After Treatment (CARAT) study, ${ }^{17,18}$ aneurysm retreatment after 1 year was more frequent in aneurysms treated with coil embolization than for those that were clipped. The annual hemorrhage rate was $0.11 \%$ per year for coil embolization, and no reruptures occurred in the surgical population. The CARAT study, however, had differences in the 2 groups of patients evaluated, and a mean follow-up of 4.4 years for clipped aneurysms and 3.7 years for coiled aneurysms. The CARAT study showed that both surgical clipping and endovascular treatment are highly effective in preventing recurrent bleeding from ruptured aneurysms. ${ }^{19}$ We gathered results in ruptured and unruptured aneurysms to determine whether there was a difference between these 2 categories. We hypothesized that ruptured aneurysms were initially less packed to avoid complications such as rupture during the procedure. When we analyzed our results, however, there was no difference in the classification of occlusion between ruptured and unruptured aneurysms. Therefore, we concluded that all aneurysms were treated the same way. The only difference we observed was the rate of retreatment for initially ruptured aneurysms and unruptured aneurysms. We retreated 46 initially ruptured aneurysms and 16 unruptured aneurysms. If coil compaction with recanalization was observed, retreatment was indicated more for patients who had bled before. The spontaneous evolution of recanalized aneurysms outweighs the procedural risks. There were minimal cases of rebleeding at follow-up, but all physicians recognize the danger of rebleeding due to its high mortality. ${ }^{12,13,17,18,20}$ Therefore, to minimize this risk, we elected to retreat all recanalization. 


\section{Retreatment}

Late retreatment is an objective end point when comparing this series to other published reports. Rates of aneurysm recurrence after coiling have been provided in various single and multicenter studies and ranged from $4.5 \%$ to $33 \%$. $^{5-9,13,16,17,19,21,22}$ In our series, 62 recanalized aneurysms were treated several times, for a total of 72 new procedures. Most, 66\%, were retreated during the first 18 months posttreatment. Considering all procedures, we found the rate of retreatment was $6 \%$ in this cohort of patients, with only $6.6 \%$ of the total treated population having been lost to follow-up. We reviewed 402 aneurysms at 5 years posttreatment, which represents $44 \%$ of the initial population of treated patients. It is very important to follow all patients regularly to better assess the time posttreatment at which an aneurysm is most susceptible to reopening. Most morphologic losses or recurrences are early, within the first year after treatment. ${ }^{16}$ In our series, $>50 \%$ of the aneurysm retreatments were performed within the first year, and 65\%, within the 18 first months.

In the publication of Campi et $\mathrm{al}^{21}$ which compares frequency, timing, and consequences of aneurysm recurrence in the ISAT population, late retreatment was 6.9 times more likely after endovascular treatment. The mean interval to retreatment was 20.7 months, with a median of 13.7 months, which is similar to that in our series. We agree that after the first year, a proportion of aneurysm remnants remains stable and is unlikely to grow and rebleed. ${ }^{16,21} \mathrm{We}$ also found a higher frequency of recurrences in the posterior communicating artery and the anterior communicating artery aneurysms, which is similar to the findings of Campi et al. ${ }^{21}$ These aneurysms are more proximal to the supra-aortic vessel and, therefore, change with variation of vascular pressure.

Angiographic and MRA follow-up are necessary during the first 2 years to detect and retreat early recanalization..$^{9-11}$ After 5 years, we found de novo aneurysms in the same places as the initially treated aneurysms. Holmin et al, ${ }^{16}$ who published the results of a monocentric study with long-term clinical outcome data and a 4- to 5-year angiographic follow-up in 466 aneurysms, found 3 de novo aneurysms and performed 90 retreatments, representing $15.2 \%$ of all procedures. They found that a stable morphologic result during the first-year interval is a good predictor of a low risk of recurrence, and we agree with this hypothesis. Long-term follow-up is necessary to identify the presence of new aneurysms and late recurrences. There appears to be no safe time limit for follow-up patients treated endovascularly. ${ }^{21}$

With regard to new aneurysms, it is very difficult to determine if they are true de novo aneurysms or a late recurrence. How does one explain this growth in the same location but not observed until 6-7 years posttreatment? Is the de novo a real aneurysm or simply a late compaction of coils due to modification of pressure? Late recurrences do occur, and this may be due to initial bad visualization of the neck of the aneurysms or due to late modification of the circulation and pressure secondary to vessel occlusion. Sometimes, it is a new aneurysm near the location of the initially treated aneurysm with an intact wall between. The same observation was made in a recent surgical study. ${ }^{23}$ Sprengers et al $^{11,20}$ found an incidence of de novo aneurysms in $1.5 \%$ of patients after 5 years. De novo aneurysms are extremely rare, but we should continue to fol- low all patients to proactively detect these malformations. It is certainly difficult to propose only observational follow-up without action, especially in patients with an initial history of subarachnoid hemorrhage. There is also a psychological aspect of follow-up to be considered, and both patients and their physicians value the importance of regular consultations during the first years after hemorrhage. ${ }^{12,19,20,23}$

With regard to the device material used, these results are interesting because at the time of our study, only bare platinum coils were used without intracranial stents or modified coils. In $10 \%$ of all procedures, however, we did use the balloon remodeling technique. Stability of occlusion was obtained in $75 \%$ of all aneurysms, and spontaneous amelioration was identified in $11 \%$ of all aneurysms. With this classic material, we obtained very good occlusion results. More than 10 years after the introduction of endovascular treatment, safety and satisfactory occlusion with bare platinum coils have been established. It is, however, important to develop new technology to investigate whether outcomes can be improved, but bare coils are used as a reference to treat standard aneurysms. New technologies such as modified coils and/or intracranial stents could be necessary for the $10 \%-15 \%$ of aneurysms that recanalize easily and for very large aneurysms. Results of these new devices, however, are sometimes disappointing, and it remains to be proved that modified coils provide better results. $^{24-26}$

One of the limitations in our study, which began in 1998, is the absence of an independent core laboratory to analyze our results. However, in each of the 5 participating centers in our study, the physician who collected and reviewed all of the aneurysms was independent of the treating physician. We did lose access to some patients with time, which resulted in a loss of available follow-up in $6.6 \%$ of all patients treated due to patient death or other medical reasons, patients moving, or failure to respond to requests for clinical and imaging followup. In a multicentric study such as ours, with such a large patient population, it is difficult to achieve compliance of follow-up in 974 patients, despite considerable effort to re-contact missing patients. Finally, we believe that we had a good rate of follow-up, and our results were objective.

In comparison with other series, to our knowledge, there are few with an equivalent extent of long-term follow-up; however, some may not yet be published. Our results are comparable with the publication of Holmin et al. ${ }^{16}$ Patient selection was similar and their follow-up was comparable, yet with more retreatments overall. In our country, EVT has existed since 1994, yet at the beginning, there were only a few select centers that performed this treatment. In 1998, before the published results of ISAT, we continued to develop this new technique and primarily treated ruptured aneurysms. It was more difficult to treat unruptured aneurysms because neurosurgeons preferred to operate on these patients, and open surgery was still very much the accepted mode of treatment for this type of aneurysm and for middle cerebral artery aneurysms. In 2009, a far broader scope of aneurysms is endovascularly treated and only very difficult aneurysms are surgically treated. EVT is considered safe, and the development of other new devices is improving the feasibility of EVT. We believe that all physicians agree with the importance of long-term 
follow-up of their patients and the consideration of follow-up beyond 5 years is warranted.

\section{Conclusions}

Our multicenter study confirmed the stability and efficacy of endovascular treatment for cerebral aneurysms with bare platinum coils. Long-term follow-up resulted in a retreatment, rate of $<7 \%$ and a rate of rebleeding $<1 \%$. The occlusion was stable in $86 \%$ all of aneurysms. Recurrence typically appeared during the first year after treatment, and retreatments occurred during the first 18 months. A proportion of aneurysm remnants remained stable. After 5 years, we observed de novo aneurysms in only 5 patients.

\section{Acknowledgment}

We thank Signe Houghton for reviewing the paper.

\section{References}

1. Guglielmi G, Vinuela F, Dion J, et al. Electrothrombosis of saccular aneurysms via endovascular approach. II. Preliminary clinical experience. J Neurosurg 1991;75:8-14

2. Guglielmi G, Vinuela F, Duckwiler G, et al. Endovascular treatment of posterior circulation aneurysms by electrothrombosis using electrically detachable coils. J Neurosurg 1992;77:515-24

3. Molyneux A, Kerr R, Stratton I, et al. International Subarachnoid Aneurysm Trial (ISAT) of neurosurgical clipping versus endovascular coiling in 2143 patients with ruptured intracranial aneurysms: a randomised trial. Lancet 2002;360:1267-74

4. Fernandez Zubillaga A, Guglielmi G, Vinuela F, et al. Endovascular occlusion of intracranial aneurysms with electrically detachable coils: correlation of aneurysm neck size and treatments results. AJNR Am J Neuroradiol 1994;15:815-20

5. Cognard C, Pierot L, Boulin A, et al. Intracranial aneurysms: endovascular treatment with mechanical detachable spirals in $\mathbf{6 0}$ aneurysms. Radiology 1997;202:783-92

6. Cognard C, Weill A, Castaings L, et al. Intracranial berry aneurysms: angiographic and clinical results after endovascular treatment. Radiology 1998;206: 499-510

7. Cognard C, Weill A, Spelle L, et al. Long-term angiographic follow-up of 169 intracranial berry aneurysms occluded with detachable coils. Radiology 1999; 212:348-56

8. Raymond J, Guilbert F, Weill A, et al. Long-term angiographic recurrences after selective endovascular treatment of aneurysms with detachable coils. Stroke 2003;34:1398-403

9. Pierot L, Delcourt C, Bouquigny F, et al. Follow-up of intracranial aneurysms selectively treated with coils: prospective evaluation of contrast-enhanced MR angiography. AJNR Am J Neuroradiol 2006;27:744-49

10. Cottier JP, Bleuzen-Couthon A, Gallas S, et al. Follow-up of intracranial aneu- rysms treated with detachable coils: comparison of plain radiographs, 3D time-of flight MRA and digital substraction angiography. Neuroradiology 2003;45:818-24

11. Sprengers ME, Van Rooij WJ, Sluzewski M, et al. MR angiography follow-up 5 years after coiling: frequency of new aneurysms and enlargement of untreated aneurysms. AJNR Am J Neuroradiol 2009;30:303-07

12. Aikawa H, Kazekawa K, Nagata S, et al. Rebleeding after endovascular embolization of ruptured cerebral aneurysms. Neurol Med Chir (Tokyo) 2007;47: 439-47, discussion 446-47

13. Gallas S, Pasco A, Cottier JP, et al. A multicenter study of 705 ruptured intracranial aneurysms treated with Guglielmi detachable coils. AJNR Am J Neuroradiol 2005;26:1723-31

14. Gallas S, Drouineau J, Gabrillargues J, et al. Feasibility, procedural morbidity and mortality, and long-term follow up of endovascular treatment of 321 unruptured aneurysms. AJNR Am J Neuroradiol 2008;29:63-68

15. Quadros RS, Gallas S, Noudel R, et al. Endovascular treatment of middle cerebral artery aneurysms as first option: a single center experience of 92 aneurysms. AJNR Am J Neuroradiol 2007;28:1567-72

16. Holmin S, Krings T, Ozanne A, et al. Intradural saccular aneurysms treated by Guglielmi detachable bare coils at a single institution between 1993 and 2005: clinical long-term follow-up for a total of 1810 patient-years in relation to morphological treatments results. Stroke 2008;39:2288-97

17. CARAT investigators. Rates of delayed rebleeding from intracranial aneurysms are low after surgical and endovascular treatment. Stroke 2006;37:143742. Epub 2006 Apr 20

18. Johnston SC, Dowd CF, Higashida RT, et al. Predictors of rehemorrhage after treatment of ruptured intracranial aneurysms: the Cerebral Aneurysm Rerupture After Treatment (CARAT) study. Stroke 2008;39:120-25. Epub 2007 Nov 29

19. Redekop GJ. Microsurgical clipping or endovascular coiling for ruptured cerebral aneurysms. Stroke 2006;37:1352-53

20. Sprengers M, Schaafsma J, Van Rooij WJ, et al. Stability of intracranial aneurysms adequately occluded 6 months after coiling: a 3T MR angiography multicenter long-term follow-up study. AJNR Am J Neuroradiol 2008;29:1768-74

21. Campi A, Ramzi N, Molyneux AJ, et al. Retreatment of ruptured cerebral aneurysms in patients randomized by coiling or clipping in the International Subarachnoid Aneurysm Trial (ISAT). Stroke 2007;38:1538-44

22. Murayama Y, Nien YL, Duckwiler G, et al. Guglielmi detachable coil embolization of cerebral aneurysms: 11 years' experience. J Neurosurg 2003;98: 959-66

23. Van der Schaaf IC, Velthuis BK, Wermer MJ, et al. New detected aneurysms on follow-up screening in patients with previously clipped intracranial aneurysms: comparison with DSA or CTA at the time of SAH. Stroke 2005;36:1753-58

24. Piotin M, Spelle L, Mounayer C, et al. Intracranial aneurysms: treatment with bare platinum coils - aneurysm packing, complex coils, and angiographic recurrence. Radiology 2007;243:500-08

25. White PM, Raymond J. Endovascular coiling of cerebral aneurysms using bioactive or coated-coil technologies: a systematic review of the literature. AJNR Am J Neuroradiol 2009;30:219-26

26. Pierot L, Bonafé A, Bracard S, et al. Endovascular treatment of intracrania aneurysms with Matrix detachable coils: immediate posttreatment results from a prospective muticenter registry. AJNR Am J Neuroradiol 2006;27: 1693-99 